\title{
High-performance transparent conducting films of long single- walled carbon nanotubes synthesized from toluene alone
}

\author{
Er-Xiong Ding, Aqeel Hussain, Saeed Ahmad, Qiang Zhang $(\bowtie)$, Yongping Liao, Hua Jiang, and Esko \\ I. Kauppinen $(\square)$
}

Department of Applied Physics, School of Science, Aalto University, Puumiehenkuja 2, 00076 Aalto, Espoo, Finland

(c) The Authors 2019

Received: 18 October 2019 / Revised: 19 November 2019 / Accepted: 25 November 2019

\begin{abstract}
Single-walled carbon nanotube (SWCNT) transparent conducting films (TCFs) are attracting increasing attention due to their exceptional optoelectronic properties. Toluene is a proposed carbon source for SWCNT synthesis, but the growth parameters of SWCNTs and their TCF optoelectronic performance (i.e., sheet resistance versus transmittance) have been insufficiently evaluated. Here, we have for the first time reported a systematic study of the fabrication of high-performance SWCNT TCFs using toluene alone as the carbon source. The mechanisms behind each observed phenomenon were elucidated using optical and microscopy techniques. By optimizing the growth parameters, high yields of SWCNT TCFs exhibiting a considerably low sheet resistance of $57 \Omega /$ sq at $90 \%$ transmittance were obtained. This competitive optoelectronic performance is mainly attributable to long SWCNT bundles (mean length is $41.4 \mu \mathrm{m}$ ) in the film. Additionally, a chirality map determined by electron diffraction displays a bimodal distribution of chiral angles divided at $15^{\circ}$, which is close to both armchair and zigzag edges. Our study paved the way towards scaled-up production of SWCNTs for the fabrication of high-performance TCFs for industrial applications.
\end{abstract}

\section{KEYWORDS}

single-walled carbon nanotubes, transparent conducting films, toluene, floating catalyst chemical vapor deposition

\section{Introduction}

Transparent conducting films (TCFs) composed of single-walled carbon nanotubes (SWCNTs) have outstanding electrical and optical properties, and consequently have high potential for various applications, such as thin-film transistors [1], solar cells [2], organic light-emitting diodes (OLEDs) [3] and strain sensors [4]. They are candidate materials for replacing indium tin oxide (ITO) films [5]. To simply evaluate the optoelectronic performance of a TCF, the sheet resistance and film transmittance (T) at $550 \mathrm{~nm}$ are required. The sheet resistance and transmittance of a SWCNT TCF are affected by nanotube diameter [6], length [7] and crystallinity [8], as well as the diameter of the SWCNT bundle [9], film morphology [10], the amount of catalyst particles in the film [8] and the purity of semiconducting SWCNTs (s-SWCNTs) [11]. The optoelectronic performance of pristine SWCNT TCFs does not usually meet the requirements of practical device applications, which require a low sheet resistance combined with high transparency. Chemical doping of TCFs is therefore often used to decrease the sheet resistances of pristine films [12]. Systematic investigation of the growth parameters of the resulting SWCNTs is then required in order to optimize their morphological features and optoelectronic properties.

Ethanol $\left(\mathrm{C}_{2} \mathrm{H}_{6} \mathrm{O}\right)[10]$ has often been employed as the carbon source in producing SWCNTs, since this results in relatively high yield and a competitive TCF performance compared with using either carbon monoxide (CO) [6] or ethylene $\left(\mathrm{C}_{2} \mathrm{H}_{4}\right)$ [13] as the carbon source. However, further improvements in
SWCNT yields and in the optoelectronic performance of TCFs are required for large-scale applications. To balance the trade-off between yield and performance, alternative active carbon sources should be explored. Toluene $\left(\mathrm{C}_{7} \mathrm{H}_{8}\right)$ has been utilized as the carbon source for the synthesis of SWCNTs in an enhanced direct injection pyrolytic synthesis (e-DIPs) method [14] and to produce CNTs for spinning fibers [15]. Both processes are characterized by a high yield of nanotubes. More recently, using toluene and ethylene as a hybrid carbon source, Jiang et al. fabricated SWCNT TCFs with a record low sheet resistance of $25 \Omega / \mathrm{sq}$ at $90 \% \mathrm{~T}$ [16]. The results indicate that toluene may have high potential as a carbon source for the synthesis of SWCNTs at high yields and with an excellent thin film performance. The use of a hybrid carbon source in the aforementioned reports $[14,16]$, however, makes it impossible to explore the effects of each carbon source individually. Unlike ethanol, the aromatic compound toluene does not contain any oxygen, which rules out the effect of oxygen on the growth of SWCNTs and is advantageous for mechanistic studies of their chirality distribution. Comprehensive investigations of the effects of growth parameters on the morphology and structure of the SWCNTs synthesized from toluene alone are, however, lacking. Evaluation of the sheet resistance versus transmittance of SWCNT TCFs produced with toluene is of particular interest.

In this work, toluene alone was hence selected as the carbon source for the synthesis of SWCNTs for TCF fabrications. The effects of selected growth parameters (i.e., the catalyst and promoter concentrations, flow rate of the carrier gas, synthesis 
temperature, and feeding rate of the precursors) on the optoelectronic performance of SWCNT TCFs were systematically studied, and corresponding mechanism was explored by optical and electron microscopic characterizations of the SWCNTs. Two dopants, nitric acid $\left(\mathrm{HNO}_{3}\right)$ and gold chloride $\left(\mathrm{AuCl}_{3}\right)$, were selected to compare the doping level. In addition, the chiral structure of the SWCNTs was statistically studied by the electron diffraction technique.

\section{Experimental section}

\subsection{Materials}

Ferrocene (98\%) and thiophene ( $\geq 99 \%)$ were purchased from Sigma-Aldrich and toluene (99.7\%) was offered by Alfa Aesar. Gold chloride ( $\geq 99.99 \%$ trace metals basis) and acetonitrile (99.8\%) were from Sigma-Aldrich. AGA in Finland provided $\mathrm{H}_{2}$ (99.999\%) and $\mathrm{N}_{2}$ which was vaporized from liquid nitrogen and purified by an OT3-4 oxygen/moisture trap from Agilent. Membrane filters with a pore size of $0.45 \mu \mathrm{m}$ were ordered from Merck Millipore, France. All the chemicals were used as-received.

\subsection{Synthesis of SWCNTs}

First, an appropriate amount (2 wt.\%-3 wt.\%) of ferrocene and a small volume of thiophene (molar ratio of $\mathrm{Fe} / \mathrm{S}$ in the range of 1.5-3) were dissolved in $5 \mathrm{~mL}$ of toluene in a vial. The solution was then sonicated for $1 \mathrm{~min}$ and loaded into a $10 \mathrm{~mL}$ glass syringe with a Teflon plunger tip (Innovative Labor Systeme, Germany) prior to being injected $(0.8-1.6 \mu \mathrm{L} / \mathrm{min})$ with a syringe pump (NE-1000 series, New Era Pump Systems, USA). The injected solution was evaporated in a heating line at $130{ }^{\circ} \mathrm{C}$ (enabled by a heating cord and a benchtop thermocouple controller from BriskHeat, USA), and the vaporized precursors were carried by a mixture of $\mathrm{H}_{2}$ (40-70 standard cubic centimeters per minute, sccm) and $\mathrm{N}_{2}(300-500 \mathrm{sccm})$. The schematics of the floating catalyst chemical vapor deposition (FCCVD) system have been reported elsewhere [17]. The values for each growth parameter investigated are listed in Table 1. As-synthesized SWCNTs were collected at the outlet of the furnace onto a $13 \mathrm{~mm}$ membrane filter to form a thin film which can be presstransferred to a target substrate for characterizations. The transmittance of the SWCNT film was controlled by collection time.

\subsection{Optical spectroscopy characterizations}

A UV-Vis-NIR spectrometer (Agilent Cary 5000) was utilized for optical absorption measurements. Absorption and transmittance spectra were acquired from the films transferred onto quartz slides. The baseline was corrected before each measurement by placing one blank substrate in the reference beam path and another in the sample beam path. Raman spectra were recorded with a Raman spectrometer (Horiba Jobin-Yvon Labram HR 800) equipped with excitation wavelengths of 488, 633 and $785 \mathrm{~nm}$. Every Raman spectrum presented was averaged from three spectra detected by each laser. The distance between each two locations was intentionally set to be longer than the mean bundle length of SWCNTs to avoid repeated detection of same tube.

\subsection{Electron microscopy characterizations}

The film morphology and bundle length of SWCNTs were characterized with a Zeiss Sigma VP scanning electron microscope (SEM) operated at an acceleration voltage of $1 \mathrm{kV}$ using an Inlens electron detector. To observe film morphology, a SWCNT film on a membrane filter was press-transferred onto $\mathrm{SiO}_{2} / \mathrm{Si}$ substrate and densified by ethanol. SEM micrographs of isolated SWCNT bundles on the $\mathrm{SiO}_{2} / \mathrm{Si}$ substrate were collected for length measurements using ImageJ. For transmission electron microscope (TEM) observation, SWCNTs were directly deposited onto a lacey carbon-coated TEM grid. A JEOL 2200FS Double Cs-corrected TEM was employed for high-resolution imaging at $200 \mathrm{kV}$ and acquiring the electron diffraction patterns of the SWCNTs at $80 \mathrm{kV}$.

\subsection{Measurement of sheet resistances and doping of SWCNT films}

The description of the four point probe system for sheet resistance measurement can be found elsewhere [10]. Each sheet resistance data point is the average of three measurement values from different parts of the SWCNT film. For $\mathrm{HNO}_{3}$ doping, concentrated $\mathrm{HNO}_{3}$ (65 wt.\%, Sigma-Aldrich) was drop-casted onto SWCNT films on a polyethylene terephthalate (PET) substrate. The films were washed with deionized water $1 \mathrm{~min}$ later and dried by compressed air flow prior to measurement. For $\mathrm{AuCl}_{3}$ doping, $50 \mu \mathrm{L}$ of $16 \mathrm{mM}$ fresh $\mathrm{AuCl}_{3}$ solution (with acetonitrile as the solvent) was dropped onto SWCNT films on a PET substrate. One minute later, the films were washed with pure acetonitrile then dried by compressed air before sheet resistance measurements were taken. All the doping processes were carried out under ambient conditions in fume hood. $\mathrm{HNO}_{3}$ was selected as a dopant during the optimization period since this allowed SWCNT films to be doped at a constant dopant concentration. Sheet resistance values obtained after $\mathrm{HNO}_{3}$ and $\mathrm{AuCl}_{3}$ doping were compared.

\section{Results and discussion}

\subsection{Optimization of precursor solution}

The ingredients of the precursor solution were optimized based on evaluations of the optoelectronic performance of the SWCNT films. The fitted curves of sheet resistance versus transmittance show that the optimal concentration of ferrocene is $2 \mathrm{wt} . \%$ (Fig. 1(a)) and the optimized Fe/S ratio is 2.5 (Fig. 1(b)). A higher ferrocene concentration results in the presence of more catalyst nanoparticles in the deposited film. The nanoparticles absorb

Table 1 Focal parameters investigated for their effects on SWCNT synthesis, and values for each of the other five growth parameters

\begin{tabular}{|c|c|c|c|c|c|c|}
\hline Parameter investigated & Ferrocene (wt.\%) & Thiophene (Fe/S) & Temperature $\left({ }^{\circ} \mathrm{C}\right)$ & $\mathrm{H}_{2}(\mathrm{sccm}$ or vol.\%) & $\mathrm{N}_{2}(\mathrm{sccm})$ & Feeding rate $(\mu \mathrm{L} / \mathrm{min})$ \\
\hline Ferrocene & $-^{\mathrm{a}}$ & 2.5 & 1,120 & 60 or $13 \%$ & 400 & 1 \\
\hline Thiophene & 2 & $-{ }^{\mathrm{a}}$ & 1,120 & 60 & 400 & 1 \\
\hline Temperature & 2 & 2.5 & $-^{\mathrm{a}}$ & 60 & 400 & 1 \\
\hline $\mathrm{H}_{2}$ & 2 & 2.5 & 1,100 & $-{ }^{\mathrm{a}}$ & 400 & 1 \\
\hline $\mathrm{N}_{2}$ & 2 & 2.5 & 1,100 & 40 & $-^{\mathrm{a}}$ & 1 \\
\hline Feeding rate & 2 & 2.5 & 1,100 & 40 or $9 \%$ & 400 & $-^{\mathrm{a}}$ \\
\hline
\end{tabular}

a - means that the parameter value can be found in the corresponding figure in the results and discussion part. Ferrocene and thiophene were studied in Fig. 1, temperature in Fig. 3, $\mathrm{H}_{2}$ and $\mathrm{N}_{2}$ in Fig. 2, feeding rate in Fig. 4. 

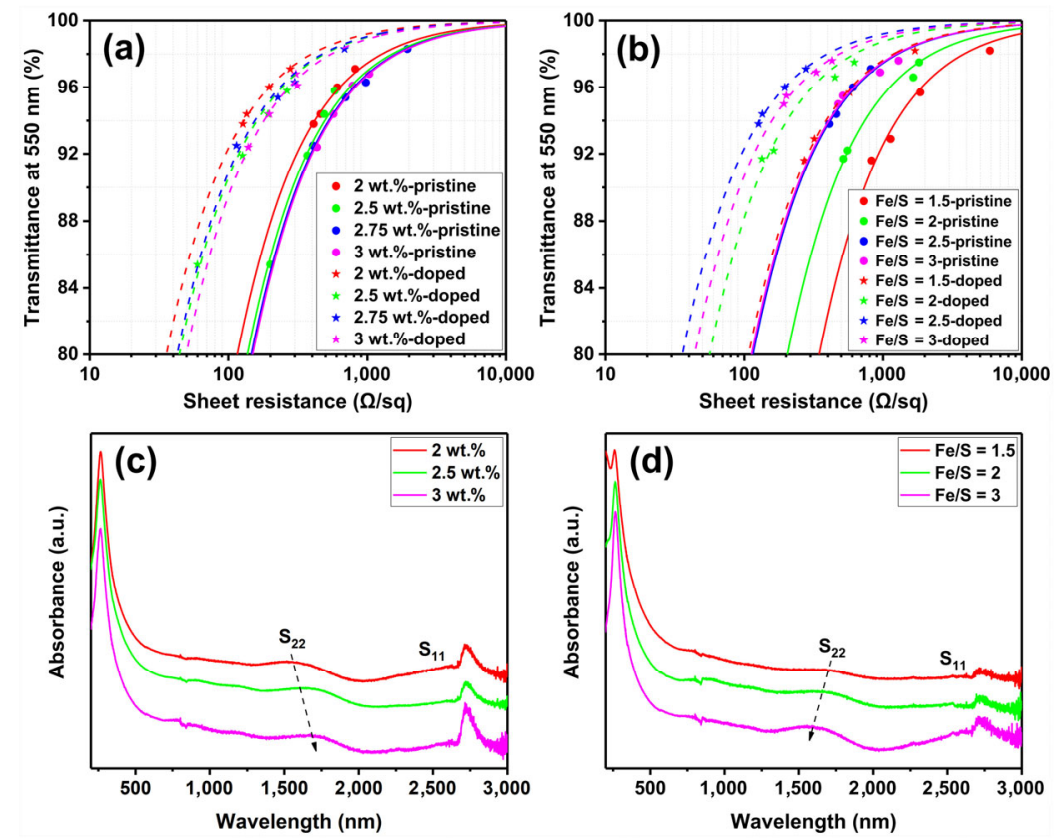

Figure 1 Optimization of the ingredients of the precursor solution by evaluating the optoelectronic performance of SWCNT films. (a) Effect of ferrocene concentration. (b) Effect of thiophene concentration. (c) and (d) are optical absorption spectra. The sharp peaks at $\sim 2,720 \mathrm{~nm}$ are ascribed to ambient absorption of $\mathrm{H}_{2} \mathrm{O}$.

more light and have a lower charge transport ability than SWCNTs. Large-diameters SWCNTs are expected with an increased ferrocene concentration (Fig. 1(c)), since SWCNT diameter is typically proportional to the size of the catalyst nanoparticle on which the SWCNT nucleates $[18,19]$. It is generally acknowledged that using an appropriate amount of sulfur in CNT synthesis can increase the yield [20], nanotube diameter [21], and even nanotube length [22], by accelerating tube nucleation and by reducing carbon encapsulation of catalyst particles [23]. Nevertheless, an excess of sulfur could induce deactivation of the catalyst due to the encapsulation of small nanoparticles with a thick layer of sulfur [8]. This leads to a non-optimal ratio of carbon to active catalyst for SWCNT growth for the fabrication of highly conductive SWCNT films. The low characteristic peaks of the first $\left(S_{11}\right)$ and second $\left(S_{22}\right)$ interband transitions of s-SWCNTs indicate an abundant background of the optical absorption spectrum with the highest sulfur concentration (lowest Fe/S molar ratio, Fig. 1(d)), implying inferior purity of the SWCNTs in the film sample. As has been reported in Refs. $[8,21,24,25]$, we also found that the mean diameter of SWCNTs increases with the concentration of thiophene. It has been proposed that the active surface area available for nanotube cap formation can be expanded via exposing more sulfur [24], which accounts for the growth of large diameter SWCNTs when an increased sulfur concentration is used. The aforementioned deactivation of some small catalyst nanoparticles at a high sulfur concentration could also reduce the formation of small-diameter tubes, resulting in the enrichment in large-diameter nanotubes.

\subsection{Effects of $\mathrm{H}_{2}$ and $\mathrm{N}_{2}$ on SWCNT yield and diameter}

In SWCNT synthesis from hydrocarbons, $\mathrm{H}_{2}$ is typically utilized as the carrier medium together with an inert gas $\left(\mathrm{N}_{2}\right.$ or $\left.\mathrm{Ar}\right)$. $\mathrm{H}_{2}$ alone as the carrier gas for SWCNT synthesis has been reported as well $[14,16]$. The introduction of $\mathrm{H}_{2}$ into a CVD reaction system generates a reducing atmosphere which inhibits the formation of carbon soot and facilitates the decomposition of the hydrocarbon precursor via hydrogen abstraction [19]. Theoretical calculations show that $\mathrm{H}_{2}$ enhances the desorption of hydrocarbons from the catalyst surface, resulting in a decrease in surface coverage and in the effective carbon supply rate [26]. We have observed that a $\mathrm{H}_{2}$ flow rate of $40 \mathrm{sccm}$ promotes the growth of high-quality SWCNTs suitable for the application of TCFs with an outstanding optoelectronic performance (Fig. 2(a)). The intensity ratio of the graphitic $\left(\mathrm{G}, \sim 1,600 \mathrm{~cm}^{-1}\right)$ and disorder-induced (e.g., catalyst particles, amorphous carbon, $\mathrm{D}, \sim 1,350 \mathrm{~cm}^{-1}$ ) bands, $I_{\mathrm{G}} / I_{\mathrm{D}}$, in a Raman spectrum has been utilized for a semi-quantitative analysis of the quality of SWCNTs. As can be seen from the $I_{\mathrm{G}} / I_{\mathrm{D}}$ ratios in Figs. $S 1(\mathrm{~d})-\mathrm{S} 1(\mathrm{f})$ in the Electronic Supplementary Material (ESM), the quality of the SWCNTs first deteriorates then improves with an increasing of $\mathrm{H}_{2}$ flow rate. This phenomenon can be explained by the formation of amorphous carbon via enhanced decomposition of toluene at a medium $\mathrm{H}_{2}$ concentration and by the gasification of amorphous carbon by hydrogen etching at a high $\mathrm{H}_{2}$ concentration $[27,28]$. Notably, the yield of carbon deposit decreases with increasing $\mathrm{H}_{2}$ flow (Fig. 2(c) and Fig. S2 in the ESM), which also occurs in our ethanol system [10, 17]. Ma et al. [27] suggested that an excess of hydrogen induced fast decomposition of the hydrocarbons (ortho-xylene and acetylene were chosen as a hybrid carbon source), resulting in the accumulation of carbon byproducts on the catalyst surface and slowing down the nanotube growth process. The suppression of the deposition of amorphous carbon at high $\mathrm{H}_{2}$ flow rates could also reduce the yield [29]. The effect of $\mathrm{H}_{2}$ on nanotube growth is relatively complicated, as it is influenced by experimental conditions (particularly carbon precursor and corresponding chemical reactions) and hence varies from system to system. In this work, $\mathrm{H}_{2}$ was found to mainly affect the nanotube yield. A more systematic study would be helpful for exploring the roles of hydrogen in the nanotube synthesis process.

As for $\mathrm{N}_{2}, 400 \mathrm{sccm}$ was found to be the optimum flow rate in terms of low sheet resistance of SWCNT films (Fig. 2(b)). Based on the absorption spectra, the mean diameter of SWCNTs shrinks when $\mathrm{N}_{2}$ flow increases (Fig. 2(d)), which is consistent with earlier results reported by Barreiro et al. [30]. This change in the mean diameter of SWCNTs can be attributed to size variation in the catalyst particles. A higher flow rate of $\mathrm{N}_{2}$ leads to a shorter 

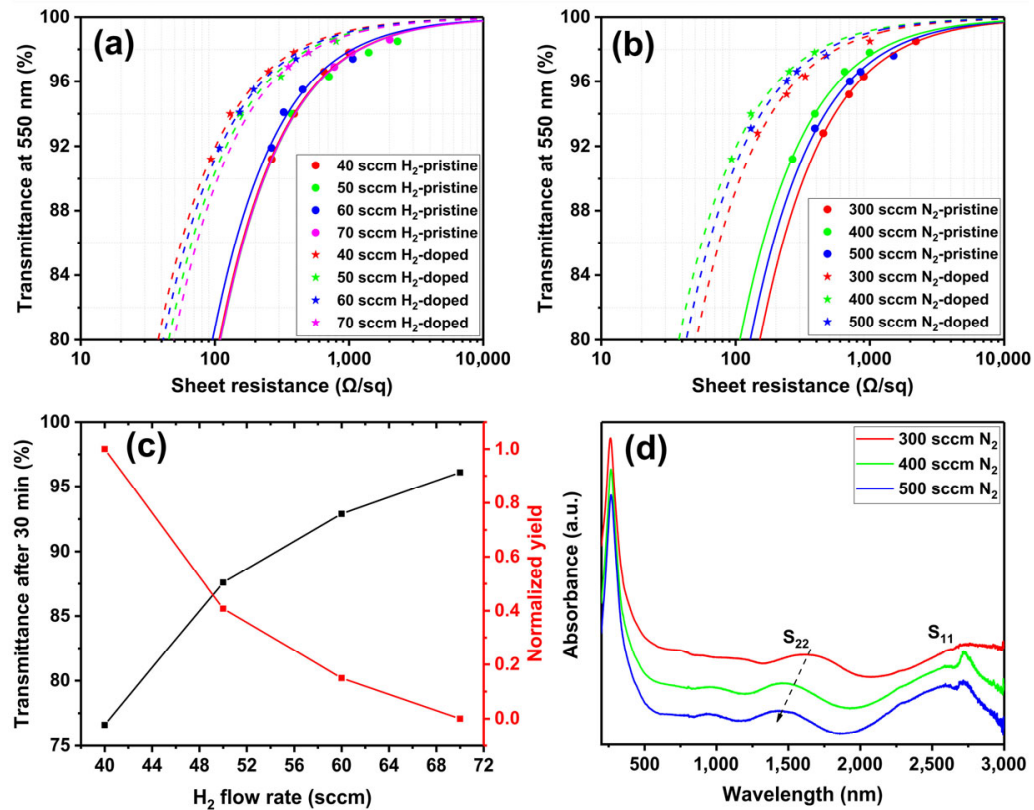

Figure 2 Effect of carrier gas. (a) and (b) are $\mathrm{H}_{2}$ and $\mathrm{N}_{2}$ effects on the optoelectronic performance of SWCNT films, respectively. (c) The dependence of deposit yield on $\mathrm{H}_{2}$ flow rate. The yield refers to absorbance and was calculated following the Beer-Lambert law. The transmittances (i.e., absorbance) after 30 min were obtained from the fitted values (Fig. S2 in the ESM). (d) Absorption spectra.

residence time (can be found in the ESM) of aerosol particles in the hot zone, which decelerates the aggregation of particles, leading to the formation of smaller-sized catalyst particles [30]. The abundance of smaller-sized particles, in turn, promotes the growth of SWCNTs with narrower diameters at higher $\mathrm{N}_{2}$ flow rates.

\subsection{Effect of synthesis temperature on SWCNT quality}

We found that a reactor temperature of $1,100{ }^{\circ} \mathrm{C}$ produces high-quality SWCNTs for the fabrication of thin films with low sheet resistances, and hence high optoelectronic performance (Fig. 3(a)). It should be noted that the doping factor of SWCNT films decreases with increasing temperature (Fig. 3(d)). There are two potential explanations for the observed doping trend. One possibility is that there are more s-SWCNTs in the sample collected at lower temperature, leading to a larger doping factor. To confirm this idea, the optical absorption spectra of SWCNT films produced at different temperatures were compared (Fig. 3(b)). Moreover, the peak areas of $S_{22}$ and the first interband transition of metallic SWCNTs $\left(\mathrm{M}_{11}\right)$ were integrated to calculate the purity of s-SWCNTs after background subtraction [31]. Unexpectedly, the proportion of s-SWCNTs in the sample collected at the lowest temperature, $1,060{ }^{\circ} \mathrm{C}$, was the lowest (Fig. S3(a) in the ESM), although the value was only 5\% lower than that for the highest purity sample. Thus, factors other than temperature also influence the doping result.

Then, we focused on the differences in the quality and diameters of SWCNTs, as observed with a Raman spectrometer and TEM. The frequency of the radial breathing mode (RBM) peak is inversely proportional to the diameter of a SWCNT. It
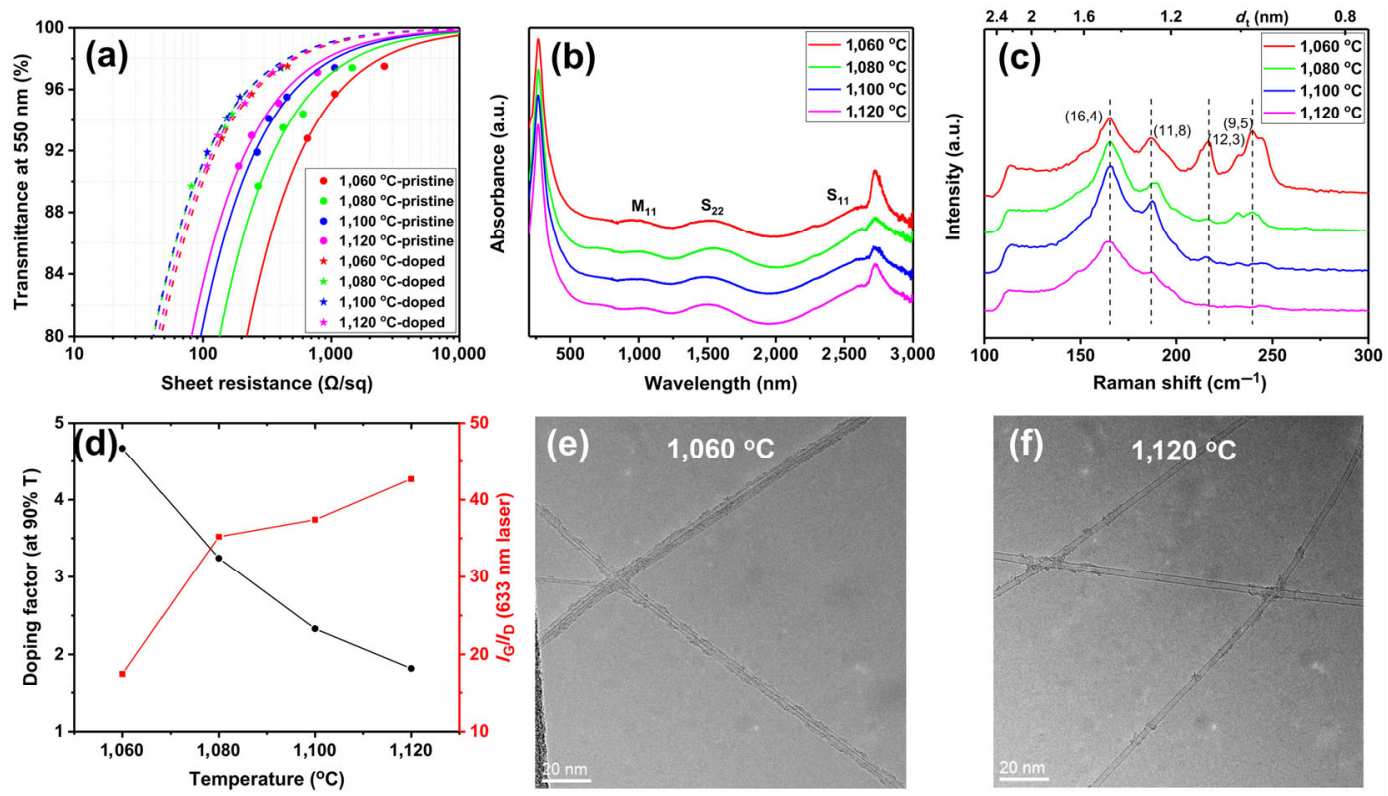

Figure 3 Effect of temperature. (a) Optoelectronic performance of SWCNT films. (b) Absorption spectra. (c) RBM peaks excited using $633 \mathrm{~nm}$ laser. (d) Temperature dependent doping factor $\left(R_{\text {pristine }} / R_{\text {doped }}\right)$ calculated at $90 \% \mathrm{~T}$ and $I_{\mathrm{G}} / I_{\mathrm{D}}$ ratio obtained with $633 \mathrm{~nm}$ laser. (e) and (f) are TEM micrographs of SWCNTs collected at 1,060 and $1,120^{\circ} \mathrm{C}$, respectively. 
turned out that more narrow-diameter SWCNTs were produced at the lowest synthesis temperature of $1,060^{\circ} \mathrm{C}$ than at high temperatures (Fig. 3(c), and Figs. S3(b) and S3(c) in the ESM). Narrow-diameter SWCNTs have wide bandgaps and their Fermi levels can be significantly shifted towards the valence band by $\mathrm{HNO}_{3}$, contributing to a large observed doping factor. The $I_{\mathrm{G}} / I_{\mathrm{D}}$ ratio increased from 17.5 to 42.7 with a temperature increase from 1,060 to $1,120^{\circ} \mathrm{C}$ (Fig. 3(d) and Fig. S3(e) in the ESM). Raman spectra of SWCNTs obtained with other excitation energies illustrate the same trend (Figs. S3(d)-S3(f) in the ESM). The charge transport ability of a SWCNT network with an inferior quality at $1,060{ }^{\circ} \mathrm{C}$ can be greatly enhanced by $\mathrm{HNO}_{3}$, which is a strong p-type dopant, via decreasing the Schottky barrier between metallic and semiconducting nanotube contact junctions [11], shifting the Fermi level towards the valence band [32], densifying the film [33], and especially removing some catalyst particles by chemical dissolving [34].

To visually verify the quality of SWCNTs collected at different temperatures, the SWCNTs synthesized at 1,060 and $1,120{ }^{\circ} \mathrm{C}$ were sampled and checked by TEM. TEM observation confirmed that the SWCNTs grown at $1,060{ }^{\circ} \mathrm{C}$ were of lower quality, presenting abundant amorphous carbon coated on the surface of nanotubes (Fig. 3(e) and Figs. S4(b)-S4(d) in the ESM) and catalyst nanoparticles in the film (Fig. S4(a) in the ESM). The SWCNTs produced at $1,120^{\circ} \mathrm{C}$ had, in contrast, a higher degree of crystallinity (Fig. 3(f) and Figs. S4(f)-S4(h) in the ESM) and fewer catalyst particles in the film (Fig. S4(e) in the ESM). The removal of more catalyst particles from the film collected at $1,060{ }^{\circ} \mathrm{C}$ and the modification of nanotube surface resulting from $\mathrm{HNO}_{3}$ treatment therefore induced more delocalized charge carriers and a larger doping factor. Based on the narrow diameters and inferior quality of the SWCNTs synthesized at $1,060{ }^{\circ} \mathrm{C}$, we tend to believe the origin is inadequate decomposition of toluene at a low temperature. Temperature is a dominant determinant of the thermal decomposition of the carbon source in the synthesis of CNTs. Reynaud et al. [8] also observed excessive soot contamination in the CNT network collected at $1,000{ }^{\circ} \mathrm{C}$, a temperature that is insufficient for the decomposition of toluene to provide enough carbon radicals for optimal SWCNTs growth. In addition, more catalyst particles might attach to the quartz tube wall at higher temperatures, though the specific mechanism behind this is unclear for the moment. Thus, a higher synthesis temperature promotes the growth of SWCNTs with higher quality. In all, the parameter window explored here is relatively narrow since we mainly focused on improving film conductivity in this work.

\subsection{Effect of feeding rate on SWCNT bundle diameter}

We have previously studied the effect of feeding rate on the optoelectronic performance of SWCNT films using ethanol as the carbon source, and found that a lower precursor feeding rate of precursors produces straight SWCNTs with narrower bundles [10]. Herein, where toluene was chosen as a new carbon source for SWCNT synthesis for the fabrication of TCFs, the feeding rate of the precursors should be also optimized to accommodate the other growth parameters.

As expected, lower feeding rates of precursors generate narrower SWCNT bundles, which is also verified by TEM observations of the bundles (Figs. 4(a) and 4(d)). The lower feeding rate of $0.8 \mu \mathrm{L} / \mathrm{min}$ produces SWCNTs with a mean bundle diameter of $6.4 \mathrm{~nm}$ (Fig. 4(b)), which reaches $8.8 \mathrm{~nm}$ at a higher feeding rate of $1.6 \mu \mathrm{L} / \mathrm{min}$ (Fig. 4(e)). Direct measurements of the electrical transport of nanotube bundles have shown that the charge carriers mainly transport at the bundle surface and that interior nanotubes only contribute to optical absorption $[35,36]$. Correspondingly, a SWCNT film composed of narrow bundles should exhibit superior optoelectronic performance. This prediction is supported by some experimental observations $[9,17,37]$. Nevertheless, we noticed here that both the pristine and doped sheet resistance values of SWCNT films collected at $0.8 \mu \mathrm{L} / \mathrm{min}$ were higher than those of films collected at $1.6 \mu \mathrm{L} / \mathrm{min}$ (Fig. 4(f)), which contrasts with our expectation based on the abovementioned reports. Therefore, we would like to propose that the relative concentrations of byproducts decomposed from toluene may have some impact on the quality of SWCNTs [19], and that this ultimately affects the optoelectronic performance of SWCNT films. We have previously reported that the feeding rate must be optimally balanced with a suitable carrier gas flow rate [10].

To examine the quality of SWCNTs collected at different feeding rates, Raman characterization was performed. The Raman results demonstrate that the quality of SWCNTs synthesized at the low feeding rate of $0.8 \mu \mathrm{L} / \mathrm{min}$ is always inferior to that of SWCNTs collected at a higher feeding rate of $1.6 \mu \mathrm{L} / \mathrm{min}$
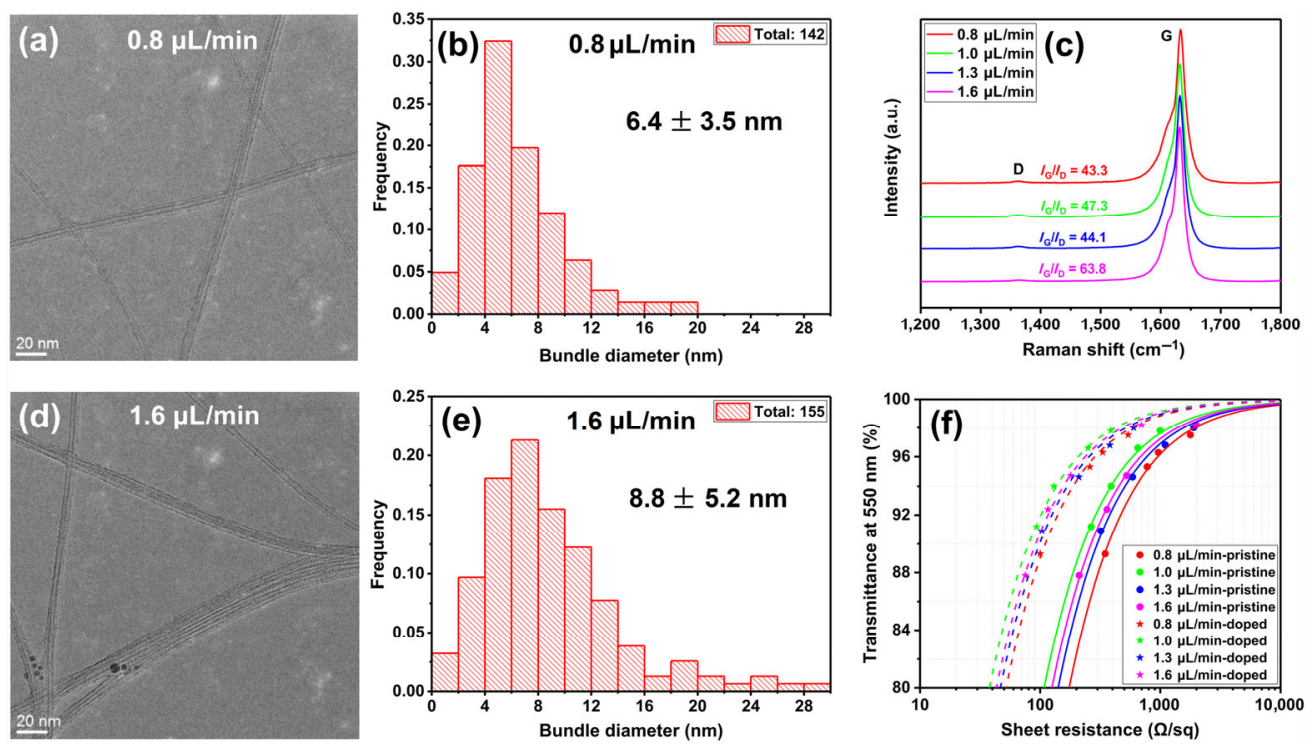

Figure 4 Effect of feeding rate. (a) and (d) are TEM micrographs of SWCNT bundles synthesized at feeding rates of 0.8 and $1.6 \mu \mathrm{L} / \mathrm{min}$, respectively. (b) and (e) are histogram distributions of the diameters of SWCNT bundles. The insert values represent the mean diameter \pm standard deviation. (c) and (f) are the feeding rate dependent $I_{\mathrm{G}} / I_{\mathrm{D}}$ ratio and optoelectronic performance of SWCNT films, respectively. 
(Fig. 4(c), and Figs. S5(d) and S5(e) in the ESM). Therefore, based on our previous experiments [10], we suggest that the flow rate of carrier gas (here both $\mathrm{N}_{2}$ and $\mathrm{H}_{2}$ ) should be slightly decreased to match with the low feeding rate of precursors for the synthesis of high-quality SWCNTs. As mentioned above, factors like the concentrations and residence times of gas phase radicals and particles could be influenced by the flow rates of $\mathrm{H}_{2}$ and $\mathrm{N}_{2}$. Additionally, as seen from the RBM peaks in Raman spectra (Figs. S5(a)-S5(c) in the ESM), more narrower-diameter SWCNTs were produced at lower feeding rates, which could be triggered by the existence of more smaller-size catalyst particles and fewer carbon radicals needed for nanotube growth, because of their reduced concentrations. Hence, it is inadvisable to claim that nanotube bundle diameter, nanotube quality, diameter or length alone contribute most to the conductivity (or sheet resistance) of a SWCNT film. A systematic experimental investigation of growth parameters assisted by big data analysis, like machine learning [38, 39], might effectively locate truly optimal conditions for producing SWCNTs for the fabrication of TCFs with an optoelectronic performance approaching or even outperforming that of ITO film.

\subsection{Measurement of SWCNT length}

SWCNT length is another critical factor affecting the sheet resistance of TCFs. SWCNTs with a sub-monolayer density were deposited onto a silicon substrate to gather SEM micrographs for length measurement (Fig. 5(a)). The mean length of SWCNT bundles was $41.4 \mu \mathrm{m}$ (thus the growth rate was estimated to be ca. $7.3 \mu \mathrm{m} / \mathrm{s}$ ) with a standard deviation of $21.5 \mu \mathrm{m}$ (Fig. 5(b)). The growth of long SWCNTs may relate to their fast growth speeds enabled by instant carbon feeding from toluene. Compared with the lengths of SWCNTs grown from carbon monoxide [6], ethylene [13] or ethanol [10] as the carbon source, we suggest that toluene, as a more active hydrocarbon, could provide enough carbon atoms for nanotube growth prior to the deactivation of the catalyst. The lengths of SWCNTs synthesized from carbon monoxide are, for instance, typically shorter than $10 \mu \mathrm{m}[6,7,9,37]$, owing to the slow decomposition of carbon monoxide, which results in an inadequate feeding rate of carbon atoms for nanotube growth. Contact junctions tend to augment network resistance. SWCNT networks comprising long SWCNTs have a reduced number of contact junctions resulting in low sheet resistance, and are for this reason preferred in TCF applications. A SEM micrograph of a representative SWCNT film surface morphology is displayed in Fig. S6 in the ESM, presenting long, straight and narrowly bundled nanotubes.

\subsection{Dopant effect and comparison of performance and yield with reported data}

As was mentioned earlier, the as-prepared SWCNT TCFs must be doped to enhance their optoelectronic performance for application purposes. At the optimization stage, all the abovementioned SWCNT films were doped with $\mathrm{HNO}_{3}$ (65 wt.\%) throughout the parameter optimization stage. To compare the doping levels of the two dopants, a $16 \mathrm{mM}$ fresh $\mathrm{AuCl}_{3}$ solution (with acetonitrile as the solvent) was utilized. In order to identify the source of the difference in doping ability, the pristine and doped films were carefully analyzed by Raman spectroscopy. The suppression of RBM peaks observed both for metallic SWCNTs and s-SWCNTs, especially at high frequencies, implies the doping of narrow diameter s-SWCNTs [7] (Fig. 6(a)). Furthermore, the blue shift (to a high frequency) of the $\mathrm{G}$ band (Fig. 6(b)) reveals that both $\mathrm{HNO}_{3}$ and $\mathrm{AuCl}_{3}$ are p-type dopants which accept electrons from SWCNTs, initiating a shift in the Fermi level towards the valence band, thus enhancing the conductivity of the SWCNT film $[32,40]$. The larger shift in the $\mathrm{G}$ band observed in this work suggests $\mathrm{AuCl}_{3}$ to be the stronger dopant. Then, the dopant effect was studied by doping the films collected at optimized conditions. After $\mathrm{HNO}_{3}$ doping, the sheet resistance of SWCNT film at $90 \%$ T decreases to ca. $66 \Omega / s q$ from a pristine value of ca. $143 \Omega /$ sq (Fig. 6(c)). However, through $\mathrm{AuCl}_{3}$ treatment, the pristine value was reduced to ca. $57 \Omega /$ sq, which is one of the lowest sheet resistances recorded (at 90\% T) for SWCNT TCFs [13, 16, 41, 42]. Previously reported SWCNT TCFs fabricated by the dry method $[6,10,13,16,41-43]$ were selected for comparison with the TCFs in this work (Fig. 6(c)). Specific morphological features of the reported SWCNTs, the adopted dopant and the optoelectronic performance of the SWCNT TCFs are summarized in Table S1 in the ESM. Jiang et al. [16] reported that achieving an optoelectronic performance challenging that of ITO film would require the production of long and isolated SWCNTs with near-ohmic contact junctions among nanotubes. However, in practice, nearohmic contact junctions are hard to achieve in as-deposited SWCNT films fabricated by direct synthesis method. Nanotube junctions could, perhaps, be welded together to facilitate electron transport through post-treatment like Joule heating [44]. The SWCNT TCF yield deposited on the filter in this work was also compared with the previously reported values $[6,10,13,16]$ (Fig. 6(d)). We achieved a high yield (7.5 times higher than that of SWCNTs synthesized from ethylene [13] with a comparable sheet resistance value) and excellent optoelectronic performance simultaneously (Fig. 6(d)), as is required for the large-scale applications of SWCNT TCFs.

\subsection{Chiral structures of SWCNTs synthesized from toluene}

As well as the optoelectronic performance of SWCNT TCFs, the chiralities of the SWCNTs are of interest, since a new carbon source was used for their production. Barnard et al. [19] released the first ever chirality map of SWCNTs synthesized from toluene,
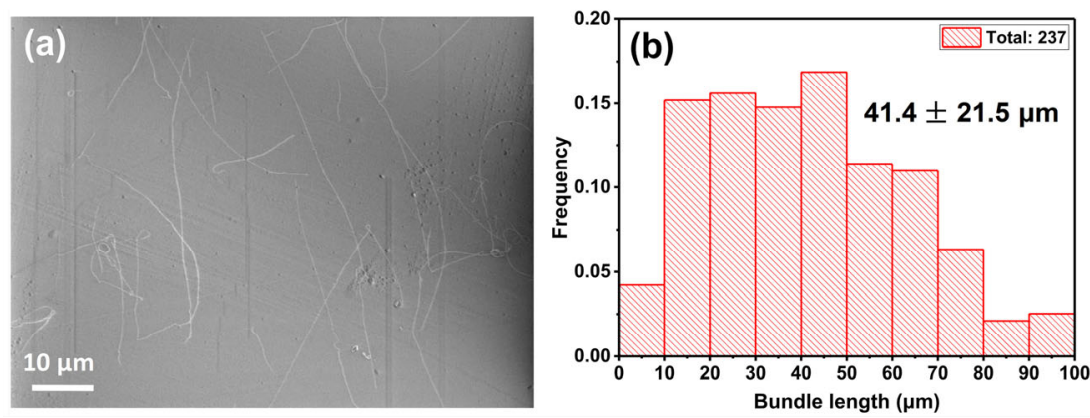

Figure 5 Study of SWCNT length. (a) SEM micrograph of isolated SWCNT bundles. (b) Histogram distribution of lengths of SWCNT bundles. Growth parameters: 2 wt.\% ferrocene, $\mathrm{Fe} / \mathrm{S}=2.5,1,100{ }^{\circ} \mathrm{C}, 40 \mathrm{sccm} \mathrm{H} \mathrm{H}_{2}, 400 \mathrm{sccm} \mathrm{N} \mathrm{N}_{2}, 1 \mu \mathrm{L} / \mathrm{min}$. 
based on the electron diffraction technique. A clearer chirality map of SWCNTs based on toluene would be useful to verify the initially reported one using a different CVD reaction system. Here, we gathered the electron diffraction patterns (Figs. S7(b) and S7(c) in the ESM) of 180 clean and isolated SWCNTs and analyzed these to depict the chirality map shown in Fig. 7(a). The chiral angles are clustered close to both armchair and zigzag edges, displaying a bimodal distribution divided roughly by the chiral angle of $15^{\circ}$ (Fig. $7(\mathrm{c})$ ). The results are consistent with those analyzed from the chirality map reported by Barnard et al. [19]. Recently, Artyukhov et al. [45] proposed a continuum model which combines free energy at the catalyst-nanotube interface and the growth barrier of a nanotube grown on solid catalyst to understand the selectivity of a chiral index. At a given diameter,

$$
A(x)=N(x) R(x) \sim x \mathrm{e}^{-x}
$$

where $A$ is the abundance of a chiral index, $N$ and $R$ are the probability and growth rate of a chiral type, respectively, $x$ is the angular deviation from the achiral direction: $x$ equals the chiral angle $\theta$ for the near zigzag type, and equals $30^{\circ}-\theta$ when close to the armchair type. A bimodal distribution of chiral angles close to both the armchair and zigzag types (see Fig. 1(f) in Ref. [45]) was then generated following this function. The calculated bimodal distribution nicely matches our experimental results on the chirality distribution (Fig. 7(a)). The free-energy barriers that must be overcome for the addition of new hexagons are considered to be lower for armchair tubes than for zigzag tubes [45], accounting for the observed enrichment $(67.8 \%)$ of near-armchair tubes. On the other hand, the interface
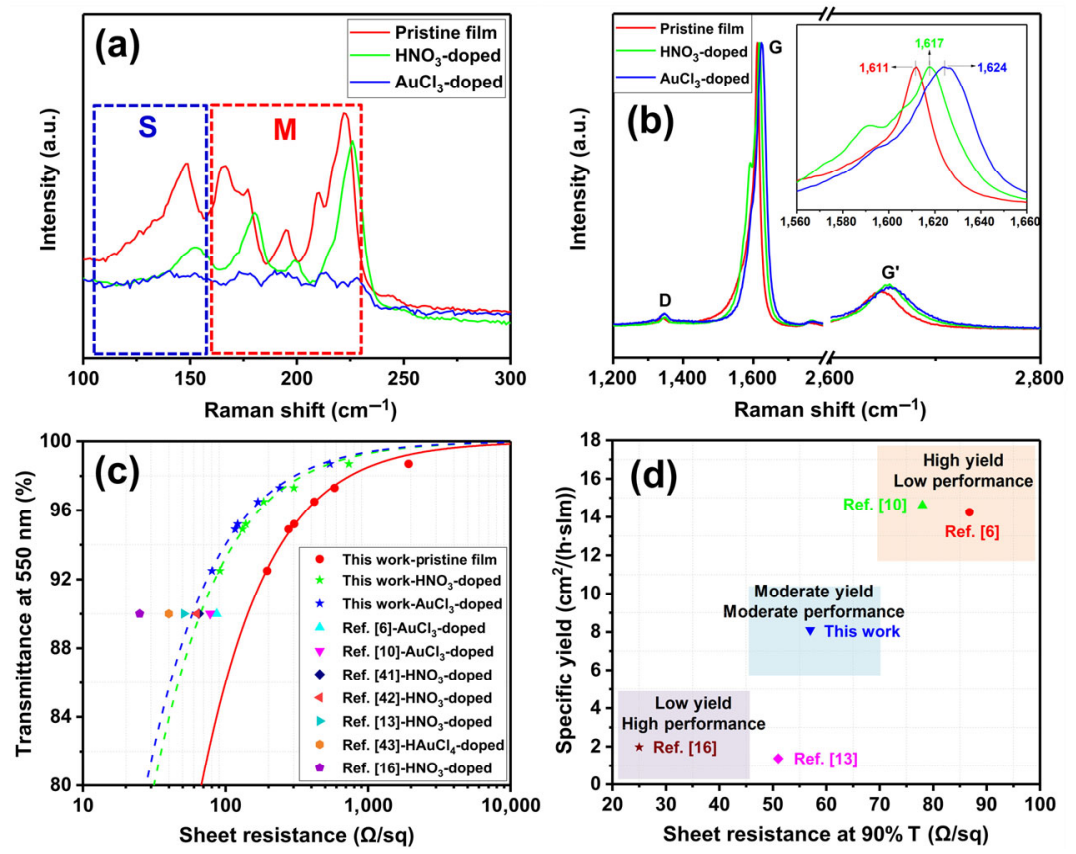

Figure 6 Dopant effect and comparison of performance and yield. (a) RBM peaks. (b) G bands. Laser wavelength is $633 \mathrm{~nm}$. The insert in (b) is the enlarged part of $\mathrm{G}$ bands to clearly show the blue shift of G band after doping. (c) Comparison of the optoelectronic performance of SWCNT TCFs. (d) Illustration of the trade-off between yield and performance. Specific yield was calculated following the formula of $\frac{\text { collection area of SWCNT film }\left(\mathrm{cm}^{2}\right)}{\text { collection time for } 90 \% \mathrm{~T} \text { film }(\mathrm{h}) \times \text { total flow }(\mathrm{slm})}$
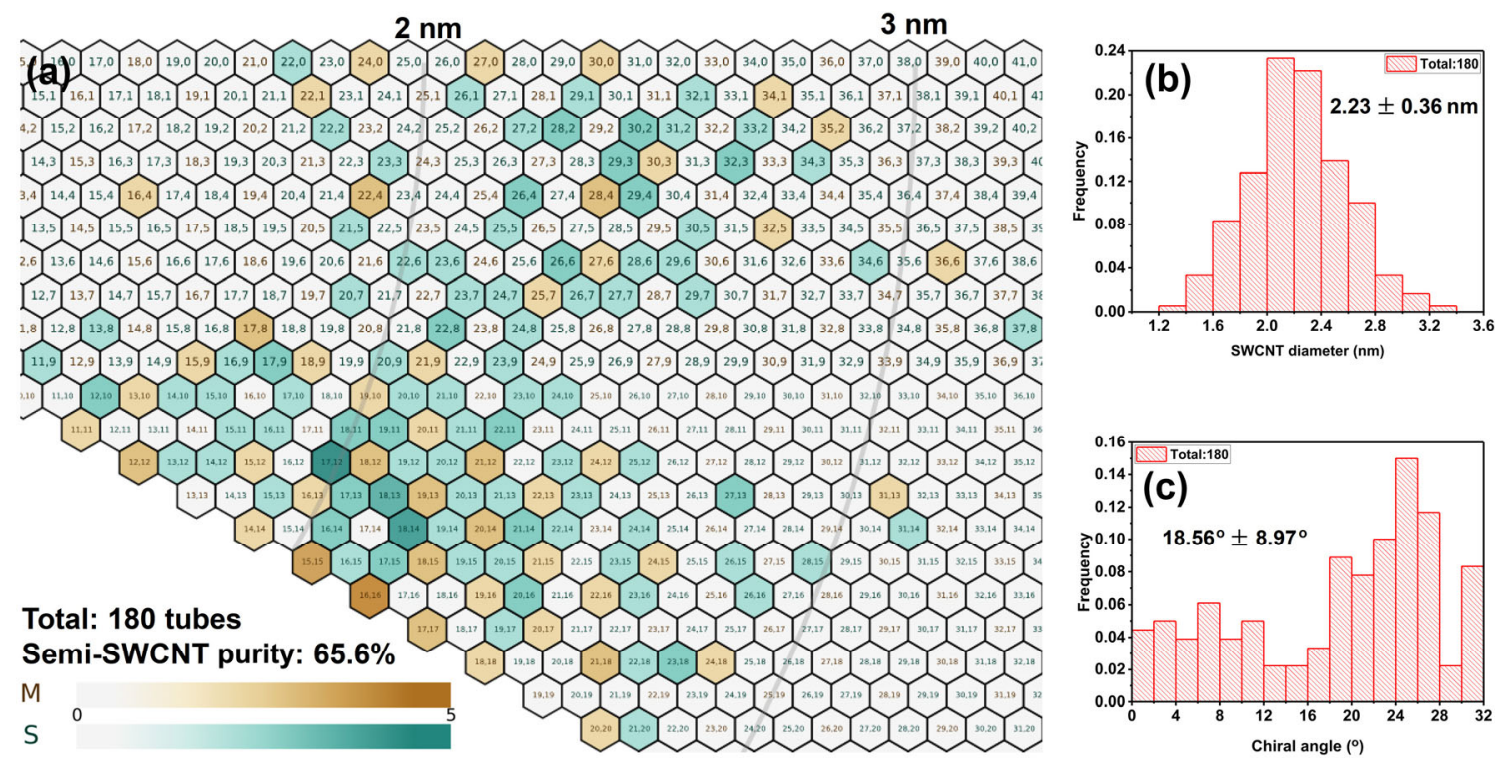

Figure 7 Study of SWCNT chiralities. (a) Chirality map determined by the electron diffraction technique. (b) and (c) are diameter and chiral angle distributions, respectively. Growth parameters: 2 wt. \% ferrocene, $\mathrm{Fe} / \mathrm{S}=2.5,1,100{ }^{\circ} \mathrm{C}, 40 \mathrm{sccm} \mathrm{H} \mathrm{H}_{2}, 400 \mathrm{sccm} \mathrm{N}, 1 \mu \mathrm{L} / \mathrm{min}$. 
energy between near-zigzag nanotubes and the catalyst surface might be reduced via a possible solid-to-liquid phase transition of the catalyst [46], when the synthesis temperature is $1,100{ }^{\circ} \mathrm{C}$, promoting the nucleation and growth of near-zigzag tubes. Thus, a bimodal distribution of SWCNT chiral angles is presented. In addition, the chirality distribution shown here suggests that the prevalence of near-armchair SWCNTs, when these are synthesized from ethanol, can be ascribed to etching by the decomposed oxygen-containing group. The fact that the purity of s-SWCNTs based on toluene is only $65.6 \%$, which is more than $10 \%$ lower than the percentage when based on ethanol, could also support the etching statement. The extraction of diameters from chiral indexs also indicates a large mean diameter of SWCNTs (ca. $2.23 \mathrm{~nm}$, Fig. 7(b)). This mean diameter matches the fitted mean diameter (ca. $2.32 \mathrm{~nm}$ ) from the absorption spectrum (Fig. S8 in the ESM). Large diameter SWCNTs benefit the conductivity of SWCNT TCFs, since numerous charge carriers can be generated by their narrow bandgaps [47]. The chirality map presented here can serve as a reference for the SWCNTs synthesized from toluene regardless of the diversity of CVD systems.

\section{Conclusion}

In summary, we have systematically investigated the growth parameters for SWCNT synthesis with toluene as the carbon source and their effects on the optoelectronic performance of SWCNT TCFs. We found that the diameters and quality of SWCNTs can be influenced by ferrocene and thiophene concentrations, the flow rate of the carrier gas, reactor temperature, and the feeding rate of precursors. The diameters of SWCNT bundles are predominantly determined by the feeding rate of the precursors. Additionally, the mean length and diameter of SWCNTs are $41.4 \mu \mathrm{m}$ and $2.3 \mathrm{~nm}$, respectively. $\mathrm{AuCl}_{3}$ was observed to be a stronger dopant. The sheet resistance of SWCNT TCF decreases to ca. $57 \Omega /$ sq, through $\mathrm{AuCl}_{3}$ treatment, from a pristine value of ca. $143 \Omega / \mathrm{sq}$ at $90 \%$ transmittance. The chiral angles of SWCNTs synthesized from toluene were found to present a bimodal distribution. Rapid fabrication of SWCNT films with quite low sheet resistances laid a solid foundation for future industrial application of SWCNT TCFs in flexible and transparent conductors.

\section{Acknowledgements}

This research received funding from projects 286546 (DEMEC) and 292600 (SUPER) supported by the Academy of Finland, as well as projects 3303/31/2015 (CNT-PV) and 1882/31/2016 (FEDOC) supported by TEKES in Finland. This work made use of the Aalto University Nanomicroscopy Center (Aalto-NMC) premises. The authors thank Dr. Mirkka Jones for proof reading.

Funding note: Open access funding provided by Aalto University.

Electronic Supplementary Material: Supplementary material (Raman spectra, collection time of SWCNT film, estimation of residence time, TEM micrographs, surface morphology of SWCNT film, comparison of SWCNT features, and electron diffraction patterns of SWCNTs) is available in the online version of this article at https:/doi.org/10.1007/s12274-0192581-7.

Open Access: This article is licensed under a Creative Commons Attribution 4.0 International License, which permits use, sharing, adaptation, distribution and reproduction in any medium or format, as long as you give appropriate credit to the original author(s) and the source, provide a link to the Creative Commons licence, and indicate if changes were made.

The images or other third party material in this article are included in the article's Creative Commons licence, unless indicated otherwise in a credit line to the material. If material is not included in the article's Creative Commons licence and your intended use is not permitted by statutory regulation or exceeds the permitted use, you will need to obtain permission directly from the copyright holder.

To view a copy of this licence, visit http://creativecommons.org/ licenses/by/4.0/.

\section{References}

[1] Sun, D. M.; Timmermans, M. Y.; Tian, Y.; Nasibulin, A. G.; Kauppinen, E. I.; Kishimoto, S.; Mizutani, T.; Ohno, Y. Flexible highperformance carbon nanotube integrated circuits. Nat. Nanotechnol. 2011, 6, 156-161.

[2] Jeon, I.; Xiang, R.; Shawky, A.; Matsuo, Y.; Maruyama, S. Singlewalled carbon nanotubes in emerging solar cells: Synthesis and electrode applications. Adv. Energy Mater. 2019, 9, 1801312.

[3] Zhang, D. H.; Ryu, K.; Liu, X. L.; Polikarpov, E.; Ly, J.; Tompson, M. E.; Zhou, C. W. Transparent, conductive, and flexible carbon nanotube films and their application in organic light-emitting diodes. Nano Lett. 2006, 6, 1880-1886.

[4] Lipomi, D. J.; Vosgueritchian, M.; Tee, B. C. K.; Hellstrom, S. L.; Lee, J. A.; Fox, C. H.; Bao, Z. N. Skin-like pressure and strain sensors based on transparent elastic films of carbon nanotubes. Nat. Nanotechnol. 2011, 6, 788-792.

[5] Yu, L. P.; Shearer, C.; Shapter, J. Recent development of carbon nanotube transparent conductive films. Chem. Rev. 2016, 116, 13413-13453.

[6] Liao, Y. P.; Hussain, A.; Laiho, P.; Zhang, Q.; Tian, Y.; Wei, N.; Ding, E. X.; Khan, S. A.; Nguyen, N. N.; Ahmad, S. et al. Tuning geometry of SWCNTs by $\mathrm{CO}_{2}$ in floating catalyst CVD for high-performance transparent conductive films. Adv. Mater. Interfaces 2018, 5, 1801209.

[7] Kaskela, A.; Nasibulin, A. G.; Timmermans, M. Y.; Aitchison, B.; Papadimitratos, A.; Tian, Y.; Zhu, Z.; Jiang, H.; Brown, D. P.; Zakhidov, A. et al. Aerosol-synthesized SWCNT networks with tunable conductivity and transparency by a dry transfer technique. Nano Lett. 2010, 10, 4349-4355.

[8] Reynaud, O.; Nasibulin, A. G.; Anisimov, A. S.; Anoshkin, I. V.; Jiang, H.; Kauppinen, E. I. Aerosol feeding of catalyst precursor for CNT synthesis and highly conductive and transparent film fabrication. Chem. Eng. J. 2014, 255, 134-140.

[9] Kaskela, A.; Laiho, P.; Fukaya, N.; Mustonen, K.; Susi, T.; Jiang, H.; Houbenov, N.; Ohno, Y.; Kauppinen, E. I. Highly individual SWCNTs for high performance thin film electronics. Carbon 2016, 103, 228-234.

[10] Ding, E. X.; Zhang, Q.; Wei, N.; Khan, A. T.; Kauppinen, E. I. High-performance single-walled carbon nanotube transparent conducting film fabricated by using low feeding rate of ethanol solution. Roy. Soc. Open Sci. 2018, 5, 180392.

[11] Blackburn, J. L.; Barnes, T. M.; Beard, M. C.; Kim, Y. H.; Tenent, R. C.; McDonald, T. J.; To, B.; Coutts, T. J.; Heben, M. J. Transparent conductive single-walled carbon nanotube networks with precisely tunable ratios of semiconducting and metallic nanotubes. ACS Nano 2008, 2, 1266-1274.

[12] Lee, J. W.; Jeon, I.; Lin, H. S.; Seo, S.; Han, T. H.; Anisimov, A.; Kauppinen, E. I.; Matsuo, Y.; Maruyama, S.; Yang, Y. Vapor-assisted ex-situ doping of carbon nanotube toward efficient and stable perovskite solar cells. Nano Lett. 2019, 19, 2223-2230.

[13] Hussain, A.; Liao, Y. P.; Zhang, Q.; Ding, E. X.; Laiho, P.; Ahmad, S.; Wei, N.; Tian, Y.; Jiang, H.; Kauppinen, E. I. Floating catalyst CVD synthesis of single walled carbon nanotubes from ethylene for high performance transparent electrodes. Nanoscale 2018, 10, 9752-9759.

[14] Saito, T.; Ohshima, S.; Okazaki, T.; Ohmori, S.; Yumura, M.; Iijima, $\mathrm{S}$. Selective diameter control of single-walled carbon nanotubes in the gas-phase synthesis. J. Nanosci. Nanotechnol. 2008, 8, 6153-6157. 
[15] Paukner, C.; Koziol, K. K. K. Ultra-pure single wall carbon nanotube fibres continuously spun without promoter. Sci. Rep. 2014, 4, 3903.

[16] Jiang, S.; Hou, P. X.; Chen, M. L.; Wang, B. W.; Sun, D. M.; Tang, D. M.; Jin, Q.; Guo, Q. X.; Zhang, D. D.; Du, J. H. et al. Ultrahighperformance transparent conductive films of carbon-welded isolated single-wall carbon nanotubes. Sci. Adv. 2018, 4, eaap9264.

[17] Ding, E. X.; Jiang, H.; Zhang, Q.; Tian, Y.; Laiho, P.; Hussain, A.; Liao, Y. P.; Wei, N.; Kauppinen, E. I. Highly conductive and transparent single-walled carbon nanotube thin films from ethanol by floating catalyst chemical vapor deposition. Nanoscale 2017, 9, 17601-17609.

[18] Nasibulin, A. G.; Pikhitsa, P. V.; Jiang, H.; Kauppinen, E. I. Correlation between catalyst particle and single-walled carbon nanotube diameters. Carbon 2005, 43, 2251-2257.

[19] Barnard, J. S.; Paukner, C.; Koziol, K. K. The role of carbon precursor on carbon nanotube chirality in floating catalyst chemical vapour deposition. Nanoscale 2016, 8, 17262-17270.

[20] Sonoyama, N.; Ohshita, M.; Nijubu, A.; Nishikawa, H.; Yanase, H.; Hayashi, J. I.; Chiba, T. Synthesis of carbon nanotubes on carbon fibers by means of two-step thermochemical vapor deposition. Carbon 2006, 44, 1754-1761.

[21] Reguero, V.; Alemán, B.; Mas, B.; Vilatela, J. J. Controlling carbon nanotube type in macroscopic fibers synthesized by the direct spinning process. Chem. Mater. 2014, 26, 3550-3557.

[22] Hoecker, C.; Smail, F.; Pick, M.; Weller, L.; Boies, A. M. The dependence of CNT aerogel synthesis on sulfur-driven catalyst nucleation processes and a critical catalyst particle mass concentration. Sci. Rep. 2017, 7, 14519.

[23] Gspann, T. S.; Smail, F. R.; Windle, A. H. Spinning of carbon nanotube fibres using the floating catalyst high temperature route: Purity issues and the critical role of sulphur. Faraday Discuss. 2014, 173, 47-65.

[24] Wei, J. Q.; Zhu, H. W.; Jia, Y.; Shu, Q. K.; Li, C. G.; Wang, K. L.; Wei, B. Q.; Zhu, Y. Q.; Wang, Z. C.; Luo, J. B. et al. The effect of sulfur on the number of layers in a carbon nanotube. Carbon 2007, 45, 2152-2158.

[25] Ren, W. C.; Li, F.; Bai, S.; Cheng, H. M. The effect of sulfur on the structure of carbon nanotubes produced by a floating catalyst method. J. Nanosci. Nanotechnol. 2006, 6, 1339-1345.

[26] Lebedeva, I. V.; Knizhnik, A. A.; Gavrikov, A. V.; Baranov, A. E.; Potapkin, B. V.; Aceto, S. J.; Bui, P. A.; Eastman, C. M.; Grossner, U.; Smith, D. J. et al. First-principles based kinetic modeling of effect of hydrogen on growth of carbon nanotubes. Carbon 2011, 49, 2508-2521.

[27] Ma, Y.; Dichiara, A. B.; He, D. L.; Zimmer, L.; Bai, J. B. Control of product nature and morphology by adjusting the hydrogen content in a continuous chemical vapor deposition process for carbon nanotube synthesis. Carbon 2016, 107, 171-179.

[28] Wang, X. K.; Lin, X. W.; Dravid, V. P.; Ketterson, J. B.; Chang, R. P. H. Carbon nanotubes synthesized in a hydrogen arc discharge. Appl. Phys. Lett. 1995, 66, 2430-2432.

[29] Liu, Q. F.; Ren, W. C.; Chen, Z. G.; Wang, D. W.; Liu, B. L.; Yu, B.; Li, F.; Cong, H. T.; Cheng, H. M. Diameter-selective growth of single-walled carbon nanotubes with high quality by floating catalyst method. ACS Nano 2008, 2, 1722-1728.

[30] Barreiro, A.; Kramberger, C.; Rümmeli, M. H.; Grüneis, A.; Grimm D.; Hampel, S.; Gemming, T.; Büchner, B.; Bachtold, A.; Pichler, T. Control of the single-wall carbon nanotube mean diameter in sulphur promoted aerosol-assisted chemical vapour deposition. Carbon 2007, $45,55-61$.
[31] Miyata, Y.; Yanagi, K.; Maniwa, Y.; Kataura, H. Optical evaluation of the metal-to-semiconductor ratio of single-wall carbon nanotubes. J. Phys. Chem. C 2008, 112, 13187-13191.

[32] Shin, D. W.; Lee, J. H.; Kim, Y. H.; Yu, S. M.; Park, S. Y.; Yoo, J. B. A role of $\mathrm{HNO}_{3}$ on transparent conducting film with single-walled carbon nanotubes. Nanotechnology 2009, 20, 475703.

[33] Geng, H. Z.; Kim, K. K.; So, K. P.; Lee, Y. S.; Chang, Y.; Lee, Y. H. Effect of acid treatment on carbon nanotube-based flexible transparent conducting films. J. Am. Chem. Soc. 2007, 129, 7758-7759.

[34] Hu, H.; Zhao, B.; Itkis, M. E.; Haddon, R. C. Nitric acid purification of single-walled carbon nanotubes. J. Phys. Chem. B 2003, 107, 13838-13842.

[35] Radosavljević, M.; Lefebvre, J.; Johnson, A. T. High-field electrical transport and breakdown in bundles of single-wall carbon nanotubes. Phys. Rev. B 2001, 64, 241307.

[36] Han, J. H.; Strano, M. S. Room temperature carrier transport through large diameter bundles of semiconducting single-walled carbon nanotube. Mater. Res. Bull. 2014, 58, 1-5.

[37] Mustonen, K.; Laiho, P.; Kaskela, A.; Susi, T.; Nasibulin, A. G.; Kauppinen, E. I. Uncovering the ultimate performance of singlewalled carbon nanotube films as transparent conductors. Appl. Phys. Lett. 2015, 107, 143113.

[38] Iakovlev, V. Y.; Krasnikov, D. V.; Khabushev, E. M.; Kolodiazhnaia, J. V.; Nasibulin, A. G. Artificial neural network for predictive synthesis of single-walled carbon nanotubes by aerosol CVD method. Carbon 2019, 153, 100-103.

[39] Khabushev, E. M.; Krasnikov, D. V; Zaremba, O. T.; Tsapenko, A. P.; Goldt, A. E.; Nasibulin, A. G. Machine learning for tailoring optoelectronic properties of single-walled carbon nanotube films. $J$. Phys. Chem. Lett. 2019, 10, 6962-6966.

[40] Kim, K. K.; Bae, J. J.; Park, H. K.; Kim, S. M.; Geng, H. Z.; Park, K. A.; Shin, H. J.; Yoon, S. M.; Benayad, A.; Choi, J. Y. et al. Fermi level engineering of single-walled carbon nanotubes by $\mathrm{AuCl}_{3}$ doping. J. Am. Chem. Soc. 2008, 130, 12757-12761.

[41] Wang, B. W.; Jiang, S.; Zhu, Q. B.; Sun, Y.; Luan, J.; Hou, P. X.; Qiu, S.; Li, Q. W.; Liu, C.; Sun, D. M. et al. Continuous fabrication of meterscale single-wall carbon nanotube films and their use in flexible and transparent integrated circuits. Adv. Mater. 2018, 30, 1802057.

[42] Mustonen, K.; Laiho, P.; Kaskela, A.; Zhu, Z.; Reynaud, O.; Houbenov, N.; Tian, Y.; Susi, T.; Jiang, H.; Nasibulin, A. G. et al. Gas phase synthesis of non-bundled, small diameter single-walled carbon nanotubes with near-armchair chiralities. Appl. Phys. Lett. 2015, 107, 013106.

[43] Tsapenko, A. P.; Goldt, A. E.; Shulga, E.; Popov, Z. I.; Maslakov, K. I.; Anisimov, A. S.; Sorokin, P. B.; Nasibulin, A. G. Highly conductive and transparent films of $\mathrm{HAuCl}_{4}$-doped single-walled carbon nanotubes for flexible applications. Carbon 2018, 130, 448-457.

[44] Yao, Y. G.; Fu, K. K.; Zhu, S. Z.; Dai, J. Q.; Wang, Y. B.; Pastel, G.; Chen, Y. N.; Li, T.; Wang, C. W.; Li, T. et al. Carbon welding by ultrafast Joule heating. Nano Lett. 2016, 16, 7282-7289.

[45] Artyukhov, V. I.; Penev, E. S.; Yakobson, B. I. Why nanotubes grow chiral. Nat. Commun. 2014, 5, 4892.

[46] Rao, R.; Pierce, N.; Liptak, D.; Hooper, D.; Sargent, G.; Semiatin, S. L.; Curtarolo, S.; Harutyunyan, A. R.; Maruyama, B. Revealing the impact of catalyst phase transition on carbon nanotube growth by in situ Raman spectroscopy. ACS Nano 2013, 7, 1100-1107.

[47] Yang, S. B.; Kong, B. S.; Jung, D. H.; Baek, Y. K.; Han, C. S.; Oh, S. K.; Jung, H. T. Recent advances in hybrids of carbon nanotube network films and nanomaterials for their potential applications as transparent conducting films. Nanoscale 2011, 3, 1361-1373. 